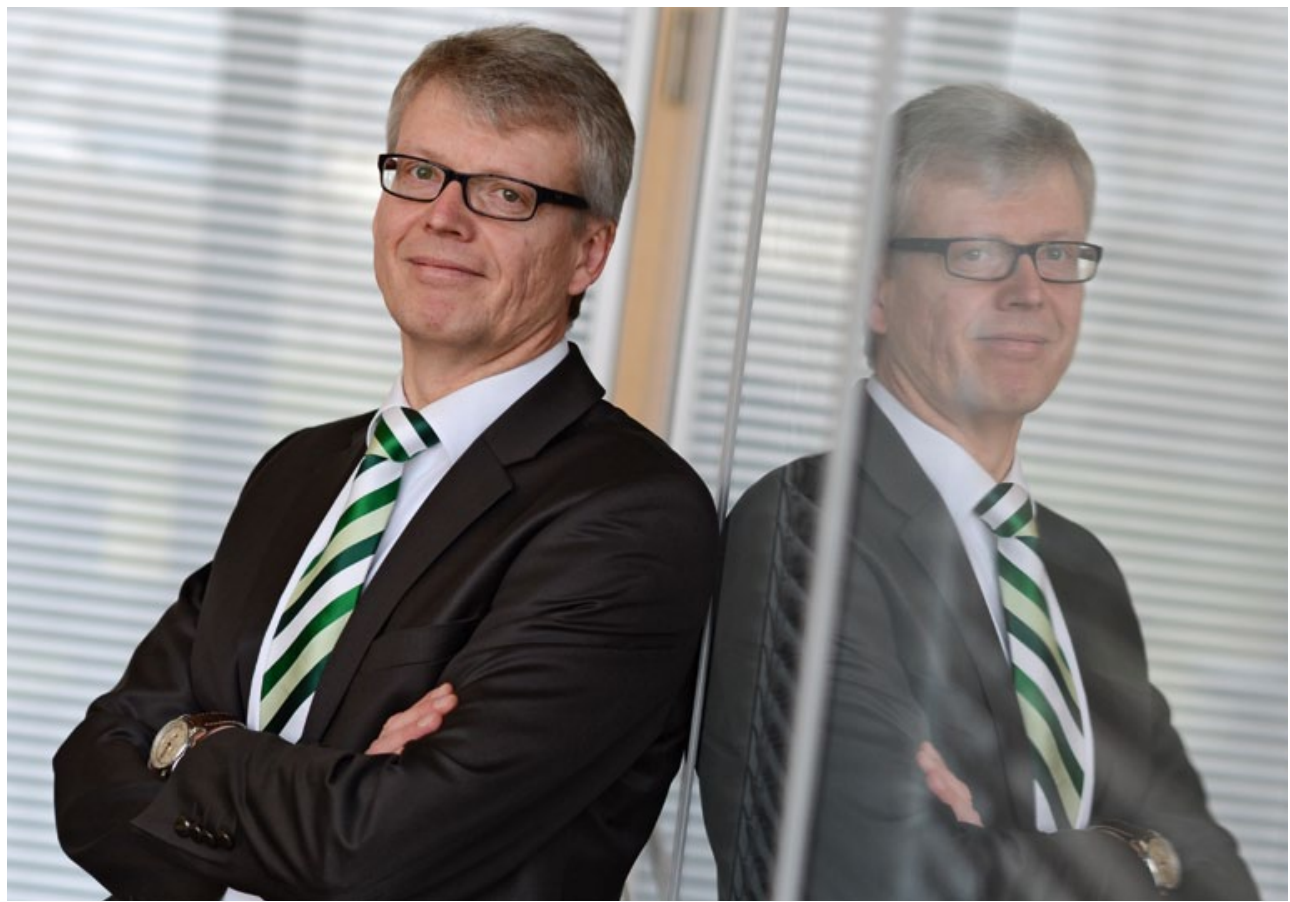

DR.-ING. HARALD NAUNHEIMER

Head of Research and Development, ZF Friedrichshafen AG

\title{
USE COMPLEXITY
}

Front-end, back-end, user interface - many end customers know these definitions from information technology but not from the automobile. In the world of software applications and softwarecontrolled electronic devices such as smartphones, tablets, or PCs, the rule - briefly summarised - holds that the full scope of a programme's functions (back-end) is concealed from the user. The user "only" sees a few defined function parameters (frontend). A defined interface, the user interface, mediates between the user's wishes and the usually very complex system.

This may sound a bit like incapacitation, but in daily life it is not - on the contrary: All of us would be overtaxed if we suddenly had to define ourselves all the functions technically available on our mobile phones or our navigation devices. With many electronic devices, we have become used to playing a supervisory role that is as demanding as it is generous: We do not have to know exactly how something works, but we have to be able to depend on it working reliably and according to our needs.

With the automobile, we are also advancing into areas of complexity which require a user interface that is oriented towards the capabilities and needs of drivers. Even today, numerous assistance systems work as a network behind the scenes. The countless, regular interventions of an adaptive damping system, for instance, are no longer noticed by the driver - it reacts within milliseconds after all. In the driveline, increasingly powerful shift strategies in automatic transmissions have significantly enhanced the original range of functions as shifting up and down. Electronic control units and energy management are now bringing the advantages of hybrid drives to the fore.

We have only just begun to realise the possibilities here. New predictive and adaptive systems will further increase safety, vehicle dynamics, and efficiency. A network between them creates additional potential. Today, we can already integrate geographical data into shift strategies. In the future, the data will be expanded to also include, for instance, traffic-related environmental information in real time. That roughly means that the control electronics, which already "knows" that the car needs to brake and downshift in a few hundred meters before a hairpin turn, will benevolently ignore the driver's request for moderate acceleration. Incapacitation? No, not if the drivers set the focal points themselves by, say, specifying a driving mode that is as economical as possible or indicating that they want to go for an especially sporty drive. This results in functions that adapt specifically to the driver and optimally assist him or her at all times. Opportunities to personalise the vehicle and its functions are a general trend. The same holds true for the individualisation of the driveline. At ZF, we can imagine for individualisation, quickly adapting software to special customer requirements via apps and updates.

Regardless of what the individual driver's request is, you can confidently transfer its optimal implementation in the specific driving situation to the mechatronic systems in the networked vehicle. 